\title{
Banco de ensayos para materiales piezoeléctricos en aplicaciones viales
}

\author{
M.VÁZQUEZ RODRÍGUEZ', F. J. JIMÉNEZ MARTÍNEZ', J. DE FRUTOS² \\ ${ }^{1}$ Dep. Sistemas Electrónicos y de Control, EUITT-UPM. Ctra. Valencia Km 728031 Madrid, España. \\ 1,2 POEMMA R\&D Group. ETSIT-UPM. Ciudad Universitaria s/n. 28040 Madrid, España.
}

\begin{abstract}
En este trabajo se define y propone un banco de ensayos que permite testar materiales piezoeléctricos para aplicaciones viales en sus diferentes configuraciones en un rango de velocidad entre los 14 y los $170 \mathrm{~km} / \mathrm{h}$, lo que cubre sobradamente las condiciones de tráfico real. Tanto el control del variador de velocidad del motor trifásico que realiza el accionamiento del banco, como la recogida de resultados de medida en las pruebas de excitación y de fatiga de los compuestos piezoeléctricos, se realiza de forma automatizada mediante código informatico. El peso que soporta cada eje del banco es configurable, permitiendo reproducir las señales obtenidas en aplicaciones donde los materiales piezoeléctricos actúan de sensores enterrados en la calzada para vehículos tanto pesados como ligeros. Gracias a esta nueva herramienta se facilita la investigación con materiales de este tipo en aplicaciones viales optimizando tanto el tipo como la disposición e instalación de los mismos, además de abrir el camino a la novedosa investigación sobre la generación y acumulación de energía eléctrica basada en materiales piezoeléctricos.
\end{abstract}

Palabras clave: Ensayo, material piezoeléctrico, sensorización vial, generación de energía

\section{Piezoelectric materials involved in road traffic applications test system}

The test bench system described in this paper performs experiments on piezoelectric materials used in road traffic applications, covering a range between 14 and $170 \mathrm{~km} / \mathrm{h}$, which is considered enough for testing under standard traffic conditions. A software has been developed to control the three phase induction motor driver and to acquire all the measurement data of the piezoelectric materials. The mass over each system's axis can be selected, with a limit of $60 \mathrm{~kg}$ over each wheel. The test bench is used to simulate the real behaviour of buried piezoelectric cables in road traffic applications for both light and heavy vehicles. This new test bed system is a powerful research tool and can be applied to determine the optimal installation and configuration of the piezoelectric cable sensors and opens a new field of research: the study of energy harvesting techniques based on piezoelectric materials.

Keywords: Test, piezoelectric material, piezoelectric characterization, road traffic, energy harvesting

\section{INTRODUCCIÓN}

Los materiales piezoeléctricos, y en especial los cerámicos, amplían su campo de aplicación de día en día. Uno de los campos en los que han entrado con mayor pujanza en los últimos años corresponde a las aplicaciones viales, donde entre otras muchas aplicaciones forman parte de los dispositivos sensores utilizados para monitorizar parámetros como la velocidad, el tipo de vehículo o la densidad de tráfico, deformación o sobrecarga de puentes, etc. (1-3). Recientemente, se ha abierto un novedoso campo de investigación en la generación y acumulación de energía eléctrica con materiales piezoeléctricos como generadores y acumuladores de carga. Como idea general, se pretende aprovechar la energía mecánica y térmica del paso de los vehículos para generar y acumular energía eléctrica (4-6). Uno de los problemas fundamentales para estos desarrollos es disponer de sistemas de modelización (7-8) y de bancos de ensayos donde someter en el laboratorio a los materiales a pruebas de comportamiento y de fatiga en condiciones equivalentes a las reales de un entorno de tráfico rodado y optimizar los procesos de generación y almacenamiento de energía. Con este objetivo, se ha diseñado un banco de ensayos versátil que permite simular las condiciones a las que se encontrarían sometidos los sensores o los dispositivos de generación de energía en régimen de uso real. Se hace uso del banco para el estudio de la generación y almacenamiento eléctrico mediante el uso de cables piezoeléctricos, analizando la respuesta de los mismos en función de la velocidad de paso y de la carga sobre cada eje para determinar la respuesta de diferentes tipos de vehículos y condiciones de rodadura de los mismos.

\section{BANCO DE ENSAYOS}

El uso de los materiales piezoeléctricos en su doble función de sensores y transductores, depende en gran medida de su ubicación, la forma en la que se ven sometidos a los estímulos y la tipología y geometría de los mismos. En cualquier caso, 
la relación entre excitación mecánica y tensión eléctrica producida en el piezoeléctrico es el principio de cualquiera de las aplicaciones del mismo. Las ecuaciones lineales que regulan este comportamiento son (9-10)

$$
\left.\left.\begin{array}{l}
T_{p}=c_{p q}^{E} S_{q}-e_{k p} E_{k} \\
D_{i}=e_{i q} S_{q}+\varepsilon_{0} \varepsilon_{i k}^{S} E_{k}
\end{array}\right\} \begin{array}{l}
S_{p}=s_{p q}^{D} T_{q}+g_{k p} D_{k} \\
E_{i}=-g_{i q} T_{q}+\left(\varepsilon_{0} \varepsilon_{i k}^{S}\right)^{-1} D_{k}
\end{array}\right\}
$$

En el caso más general, en el que el dispositivo sensor no está sometido a campos eléctricos de excitación, la densidad de carga en un material piezoeléctrico $\mathbf{D}\left(\mathrm{C} \mathrm{m}^{-2}\right)$ viene definida por la ecuación [2].

$$
\left[\begin{array}{l}
D_{x} \\
D_{y} \\
D_{z}
\end{array}\right]=\left[\begin{array}{llllll}
d_{11} & d_{12} & d_{13} & d_{14} & d_{15} & d_{16} \\
d_{21} & d_{22} & d_{23} & d_{24} & d_{25} & d_{26} \\
d_{31} & d_{32} & d_{33} & d_{34} & d_{35} & d_{36}
\end{array}\right]\left[\begin{array}{l}
\sigma_{x x} \\
\sigma_{y y} \\
\sigma_{z z} \\
\tau_{y z} \\
\tau_{z x} \\
\tau_{x y}
\end{array}\right]
$$

siendo: $\mathbf{d}=\left[\mathrm{d}_{\mathrm{ij}}\right]$ la matriz de coeficientes piezoeléctricos del material $\left(\mathrm{C} . \mathrm{N}^{-1}\right)$.

$\mathbf{T}=\left[\mathrm{T}_{\mathrm{n}}\right]$ es la matriz de tensiones mecánicas, en tracción $\sigma_{\mathrm{ij}}$ y cizalladura $\tau_{\mathrm{ij}}\left(\mathrm{N} . \mathrm{m}^{-2}\right)$

Esto pone de manifiesto las muchas posibilidades que hay tanto de excitar el material como de mejorar su respuesta y su durabilidad. Todo ello hace muy recomendable el disponer de un banco de pruebas que permita simular las condiciones de uso para la optimización de las mismas y mejorar el aprovechamiento de las propiedades del material piezoeléctrico

\subsection{Arquitectura del Sistema}

La hipótesis admitida en el diseño del banco, considera tráfico continuo, persiguiendo la generación de respuestas en los materiales idénticas a las recogidas bajo el asfalto. En esencia, el banco de pruebas está formado por una plataforma giratoria de diferentes configuraciones, accionada por un motor y controlada mediante sistema que permite la rodadura a diferentes velocidades, pesos y separaciones relativas entre ejes.

En la figura 1(a) se presenta la vista de planta de la plataforma móvil del banco de ensayos. Se ha detallado el ángulo al que se puede ajustar los dos ejes de rodadura. Tomando como origen de referencia al eje horizontal, es posible ajustar el segundo eje a $22^{\circ}, 45^{\circ}$ ó $90^{\circ}$ respecto al primero, utilizando el mecanizado realizado para la fijación de las ruedas industriales solidarias a ese eje. Las tres posibles ubicaciones del segundo eje se han representado en la propia figura 1(a). En la figura 1(b) se presenta una fotografía del banco en funcionamiento en nuestras instalaciones. Se ha procurado reproducir las condiciones dinámicas de un vehículo automóvil de tipo medio. La realización de ensayos sobre otras medidas reales es fácilmente implementable, sin más que modificar el mecanizado.

Se han seleccionado ruedas industriales con cámara de aire, con un perfil de rodadura lo más próximo a la pisada de una rueda estándar real.Para someter a los cables a excitaciones que provengan de tráfico pesado se dispone de soportes que permiten colocar pesas suplementarias, así como de unas piezas almohadilladas semirígidas que adaptan el ancho de pisada y amplifican mecánicamente el estímulo de presión.

El control del equipo variador de velocidad del motor trifásico que acciona el sistema, la captación de la velocidad real del banco de ensayos y la recogida de los datos de los experimentos se han automatizado realizando un programa en LabView ${ }^{\mathrm{TM}}$ que junto con la tarjeta de adquisición de datos DAQ6251 de National Instruments ${ }^{\mathrm{TM}}$ y un ordenador personal configuran la arquitectura de nuestro sistema tal y como reproduce la figura 2 .

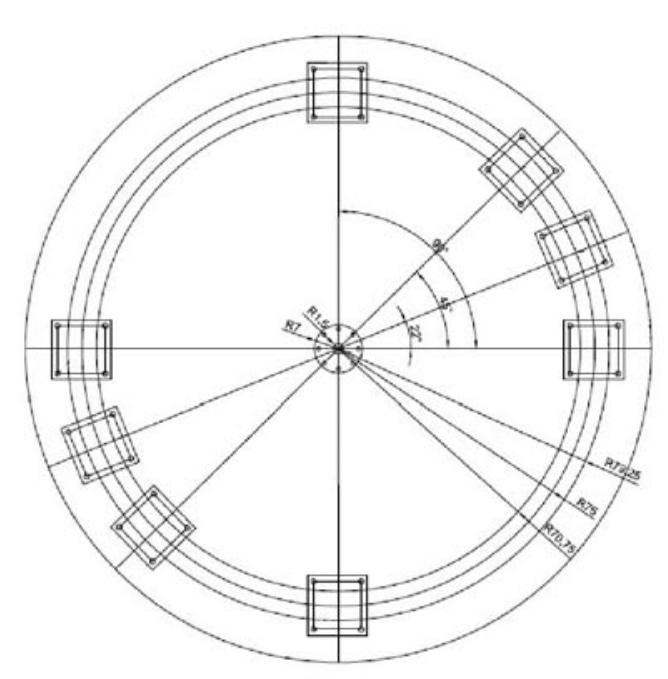

(a).

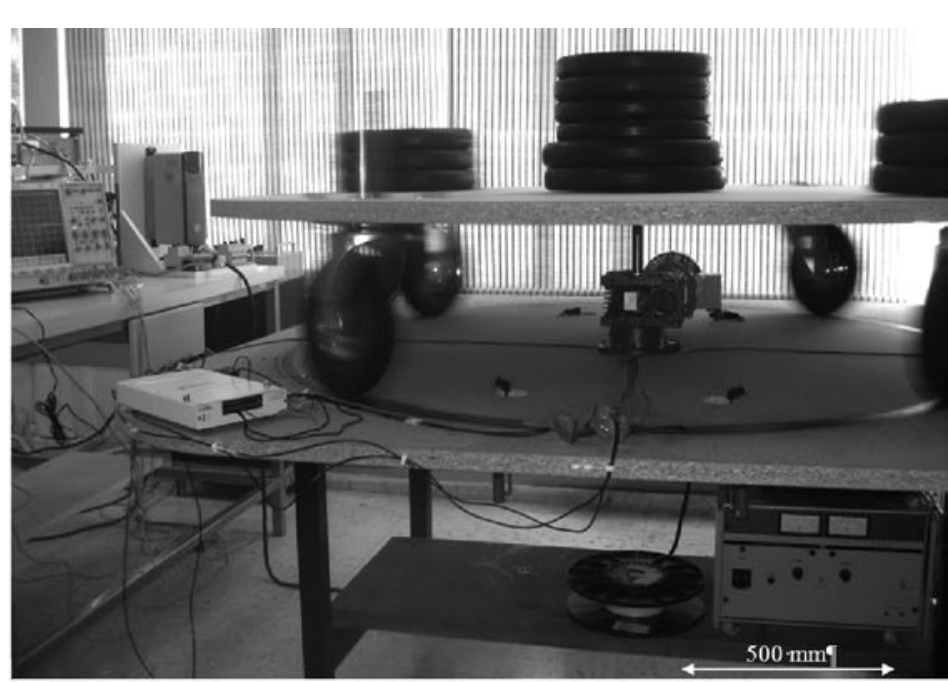

(b)

Figura 1: (a)Planta de la plataforma giratoria del banco de ensayos. (b) Ensayo en realización 
La automatización de los procesos de medida facilita el posterior tratamiento de los datos relativos al comportamiento y fatiga de los materiales. Uno de los focos de interés de nuestra investigación es obtener el modelo eléctrico real de los respectivos materiales a partir de las medidas efectuadas con el banco de ensayos, dado que resulta imprescindible si se pretende utilizar sus propiedades piezoeléctricas en el campo de la generación y acumulación de energía eléctrica.

\subsection{Prestaciones}

El banco de ensayos reproduce las condiciones de estímulo que reciben los materiales de tipo piezoeléctrico en aplicaciones viales, permitiendo por tanto realizar pruebas dónde la velocidad de las ruedas que excitan al material como la presión o deformación unitaria que se le transmite es ajustable. A continuación se describen las prestaciones que se alcanzan con el banco de ensayos diseñado desde el punto de vista cinemático e inercial.

\subsubsection{PRESTACIONES CINEMÁTICAS}

El control del tiempo entre los impactos de las ruedas de los ejes de la plataforma móvil al pasar sobre el punto donde se dispone el material piezoeléctrico sometido a ensayo permite calcular la velocidad equivalente en $\mathrm{km} / \mathrm{h}$ de un vehículo del que se conoce la distancia que separa sus ejes delantero y trasero, es decir, lo que se conoce por batalla en el campo automovilístico. El ajuste del tiempo de impacto entre ejes se logra actuando sobre el motor que acciona el banco de ensayos mediante la programación del equipo variador de velocidad que puede ajustar la frecuencia de alimentación de la tensión trifásica entre 0 y $70 \mathrm{~Hz}$.

Atendiendo a las características par-velocidad del motor y conocido el par inercial máximo aplicable, se establecen los siguientes límites prácticos para la frecuencia de la tensión alterna sinusoidal de alimentación del motor de inducción: $25 \mathrm{~Hz}$. para fijar la velocidad angular mínima y $70 \mathrm{~Hz}$ para establecer su valor máximo. Adicionalmente a la modificación de la velocidad del motor trifásico, se ha provisto al banco

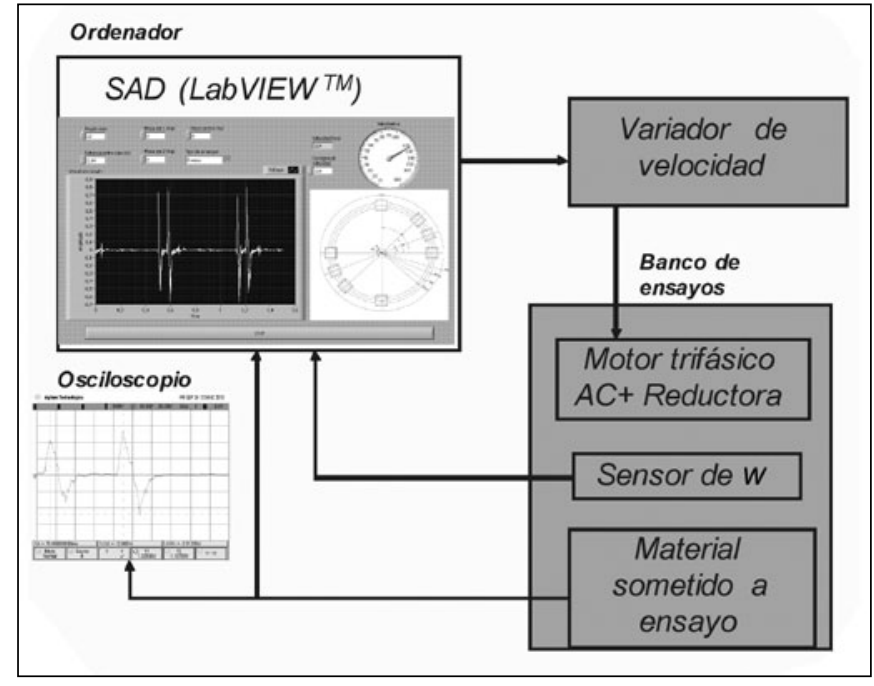

Figura 2: Arquitectura del Banco de Ensayos

de ensayos de otro grado de libertad, dado que es posible modificar el ángulo entre los dos ejes de rodadura de la plataforma, tal y como se aprecia en la figura $1(\mathrm{a})$, a $22^{\circ}, 45^{\circ} \mathrm{o}$ $90^{\circ}$, permitiendo cubrir los rangos disponibles de emulación de tráfico real que se resumen en la tabla I. En ella se detalla la velocidad equivalente para el caso de un vehículo automóvil de tipo medio, cuya separación entre ejes (batalla) sea de 2,64 m. La versatilidad del banco de ensayos es muy grande dado que el cambio de consigna de velocidad se produce en 5 segundos y el cambio de rango mediante la modificación del ángulo entre ejes, desplazando las ruedas de uno de los ejes, precisa de no más de 10 minutos de intervención.

Para adaptar tanto la velocidad de rotación de la plataforma giratoria como el par de trabajo necesario a los valores que entrega el motor, se ha acoplado una caja reductora al eje del motor. El conjunto motor-reductora permite obtener una velocidad de giro en el eje de la plataforma móvil comprendida entre 23,3 rpm y $65 \mathrm{rpm}$, que se obtiene al ajustar la frecuencia de alimentación del motor entre $25 \mathrm{~Hz}$ y $70 \mathrm{~Hz}$ respectivamente. Si se define el ángulo entre ejes como

TABLA I. RESUMEN DE PRESTACIONES DEL BANCO DE ENSAYOS: RANGO DE VELOCIDADES SIMULADAS SEGÚN VELOCIDAD DEL EJE DE SALIDA DE LA PLATAFORMA Y DEL ÁNGULO ENTRE EJES DE RODADURA.

\begin{tabular}{|c|c|c|c|c|c|c|c|c|}
\hline Ensayo & $\begin{array}{c}\text { f variador } \\
\text { AC } \\
(\mathrm{Hz})\end{array}$ & $\begin{array}{c}\text { Eje de salida } \\
n_{0}(\mathrm{rpm})\end{array}$ & $\begin{array}{c}\text { Ángulo entre } \\
\text { ejes } \\
\beta\left(\left(^{\circ}\right)\right.\end{array}$ & $\begin{array}{l}\text { Radio } \\
\text { rodadura } \\
(\mathrm{cm})\end{array}$ & $\begin{array}{c}\text { Batalla- } \\
\text { automóvil } \\
\text { berlina- } b(\mathrm{~cm})\end{array}$ & $\begin{array}{l}\text { Velocidad } \\
\text { equivalente } \\
\mathrm{v}(\mathrm{m} / \mathrm{s})\end{array}$ & $\begin{array}{l}\text { Velocidad } \\
\text { equivalente } \\
\mathbf{v}(\mathbf{k m} / \mathbf{h})\end{array}$ & $\begin{array}{c}\text { Tiempo entre } \\
\text { ejes } \\
t_{e}(s)\end{array}$ \\
\hline 1 & $70 \mathrm{~Hz}$ & 65,0 & 22 & 75 & 264 & 46,800 & 168,480 & 0,056410 \\
\hline 2 & $50 \mathrm{~Hz}$ & 46,6 & 22 & 75 & 264 & 33,552 & 120,787 & 0,078683 \\
\hline 3 & $25 \mathrm{~Hz}$ & 23,3 & 22 & 75 & 264 & 16,776 & 60,394 & 0,157367 \\
\hline 4 & $70 \mathrm{~Hz}$ & 65,0 & 45 & 75 & 264 & 22,880 & 82,368 & 0,115384 \\
\hline 5 & $50 \mathrm{~Hz}$ & 46,6 & 45 & 75 & 264 & 16,403 & 59,052 & 0,160944 \\
\hline 6 & $25 \mathrm{~Hz}$ & 23,3 & 45 & 75 & 264 & 8,201 & 29,526 & 0,321888 \\
\hline 7 & $70 \mathrm{~Hz}$ & 65,0 & 90 & 75 & 264 & 11,440 & 41,184 & 0,230769 \\
\hline 8 & $50 \mathrm{~Hz}$ & 46,6 & 90 & 75 & 264 & 8,201 & 29,526 & 0,321888 \\
\hline 9 & $25 \mathrm{~Hz}$ & 23,3 & 90 & 75 & 264 & 4,101 & 14,763 & 0,643776 \\
\hline
\end{tabular}


$\beta\left(^{\circ}\right)$, la velocidad angular de la plataforma como $\mathrm{n}_{\mathrm{o}}(\mathrm{rpm}) \mathrm{y}$ siendo $\mathrm{b}(\mathrm{cm})$ la dimensión que define la separación de los ejes delantero y trasero de un automóvil, la ecuación 3, permite obtener de forma inmediata la velocidad lineal equivalente, $\mathrm{v}$, que es posible obtener en cada ensayo, expresada en $\mathrm{km} / \mathrm{h}$.

$$
v=\frac{b}{\left(\frac{\beta}{n_{o} \cdot 6}\right) \cdot 100} \cdot 3,6
$$

En la última columna de la tabla I se calcula el tiempo expresado en segundos entre impactos de los dos ejes cuando pasan sobre el mismo punto de la plataforma fija inferior, mediante:

$$
t_{e}=\frac{\beta}{n_{o} \cdot 6}
$$

Este tiempo permite cotejar rápidamente la información registrada en las gráficas adquiridas por la aplicación automatizada o alternativamente por la imagen registrada en el osciloscopio, con la velocidad equivalente de un automóvil. La medida exacta de la velocidad equivalente se realiza mediante el bloque denominado sensor de $\omega$ en la figura 2. Este bloque está formado por varios componentes. Primeramente, un cable piezoeléctrico dispuesto sobre la plataforma estática a $90^{\circ}$ del lugar dónde se ubican los cables objeto de ensayo recoge, con cada paso de cada eje, una señal que se acondiciona posteriormente mediante un circuito electrónico diseñado para este fin. A continuación, el software de adquisición que hemos desarrollado procesa esta señal acondicionada al haberse incorporado al ordenador mediante un canal digital de entrada de la tarjeta DAQ6251. La resolución temporal del sistema de adquisición de datos es de $1 / 20 \mathrm{MHz}$, lo que permite obtener la medida de velocidad con alta exactitud. Esta medida de velocidad real realimenta el accionamiento del motor trifásico para conseguir la consigna de velocidad impuesta en cada ensayo.

\subsubsection{PRESTACIONES INERCIALES}

Para realizar los ensayos ejerciendo diferentes estímulos mecánicos sobre el material se han diseñado unos soportes para aplicar masa adicional sobre los ejes de rodadura. En la figura 1(b) se puede observar un ensayo con $160 \mathrm{~kg}$ de masa suplementaria en uno de los ejes. El diseño del soporte admite la ubicación de múltiples pesas, lo que permite modificar a voluntad el peso en cada una de las ruedas.

Adicionalmente a la masa en los extremos del eje, sobre el centro de la plataforma se pueden disponer masas adicionales hasta el límite conjunto de $200 \mathrm{~kg}$ aproximadamente tal y como se detalla seguidamente.

El elemento actuador del banco de ensayos es un motor trifásico SIEMENS modelo 1LA5083-4AA-22, cuyas características nominales son: $1 \mathrm{HP}$. de potencia eléctrica y $1500 \mathrm{rpm}$ a frecuencia de $50 \mathrm{~Hz}$. El motor se encuentra acoplado a una caja reductora MOTOVARIO ${ }^{\circledR}$ de relación 30:1, modelo NMRV63, montada a $90^{\circ}$ con relación al eje motor y cuyo eje de salida hueco se adapta al elemento de acople a la plataforma. El par motor nominal disponible en su eje se obtiene de la siguiente ecuación:

$$
\mathrm{T}_{n}=\frac{9550 \bullet P_{n}}{n}
$$

Siendo $\mathrm{P}_{\mathrm{n}}$ la potencia nominal del motor expresada en $\mathrm{kW}$ y $\mathrm{n}$ la velocidad angular del motor expresada en rpm. Al expresar las condiciones nominales se asume que la frecuencia de la tensión de alimentación del motor es de 50 Hz. Así, el motor de potencia 0,74 kW (1HP), a $1400 \mathrm{rpm}$ (velocidad real, con un deslizamiento de $100 \mathrm{rpm}$ respecto a la velocidad de sincronismo), entrega un par motor nominal de $\mathrm{T}_{\mathrm{n}}=5,05 \mathrm{Nm}$. Así pues, en el eje de salida de la reductora se podrían obtener hasta $151,5 \mathrm{Nm}$, al haber elegido para la reductora una relación 30:1, que permite abarcar el rango de velocidades prácticas recogido en la tabla I. No obstante lo anterior, las características técnicas de la reductora aconsejan para mantener un factor de servicio de la misma acorde a la aplicación que hemos diseñado que el par máximo en el eje de la reductora no exceda de $115 \mathrm{Nm}$.

El equipo variador de velocidad que alimenta y controla al motor es el modelo Dinverter DIN220075B de Control Techniques Drives Ltd. Se ha programado el control que realiza el equipo variador de velocidad con consigna de mando de tipo rampa de 5 segundos. En estas condiciones, la velocidad de entrada a la reductora de $1400 \mathrm{rpm}$ equivale a 46,67 rpm en el eje de salida de la misma, es decir una

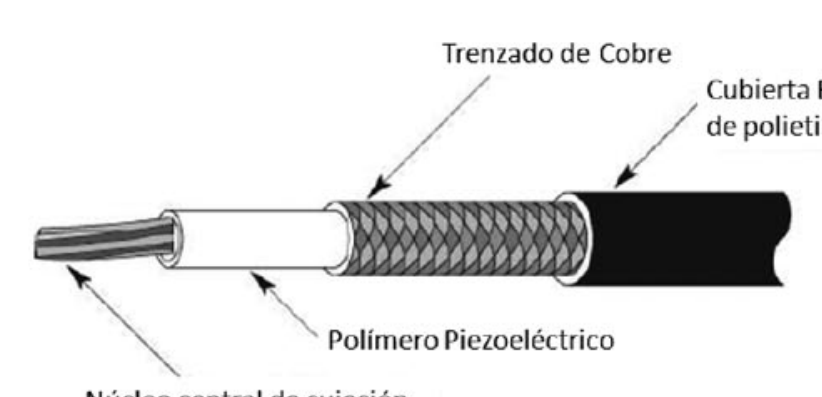

Núcleo central de sujeción

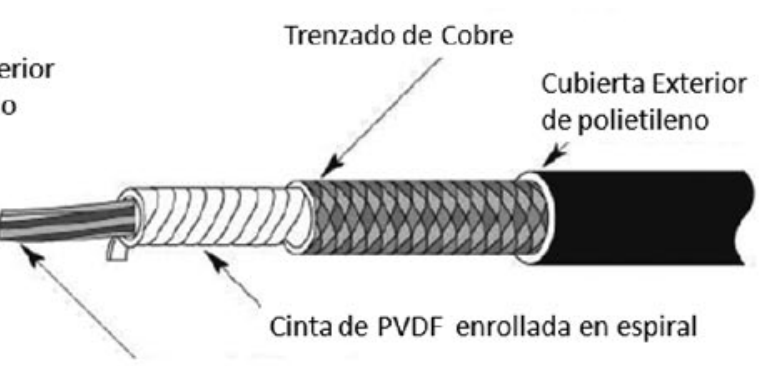

Núcleo central de sujeción 
aceleración angular de la plataforma móvil de 1,37 $\mathrm{rad} / \mathrm{s}^{2}$. Esta aceleración programada determina que, dado que el par de salida de la reductora es:

$$
\mathrm{T}_{r}=J_{T} \bullet \alpha
$$

el comportamiento inercial del banco de ensayos admite hasta un máximo de $83,94 \mathrm{Nm}^{2}$ de momento de inercia equivalente. Finalmente, una vez que la plataforma circular se ha mecanizado utilizando un tablero de material aglomerado de $30 \mathrm{~mm}$ de espesor, al que le corresponde $21,22 \mathrm{Kg} / \mathrm{m}^{2}$, y que la masa de cada rueda industrial modelo LP 261R de Blickle $^{\circledR}$ es de $4,48 \mathrm{~kg}$, permite satisfacer que el par inercial máximo aplicable nominal se consumirá con una masa suplementaria total de $202,73 \mathrm{~kg}$, límite máximo a aplicar en los ensayos a realizar.

El diseño de la pieza de acople al eje de rotación (eje de salida de la reductora) y de los soportes para masa suplementaria, la selección del variador de velocidad comercial y del conjunto motor-reductor, así como la posición óptima de este conjunto en el banco de ensayos junto con la disposición, forma geométrica y masa adecuada de las plataformas estática $\mathrm{y}$ rotatoria que lo componen, se ha realizado de forma original por este equipo de trabajo, procediéndose a todo el ensamblado de sus piezas en nuestras instalaciones.

\section{RESULTADOS EXPERIMENTALES}

A modo de ejemplo del funcionamiento del banco de ensayos anteriormente descrito, someteremos al mismo una disposición de cable piezoeléctrico, que puede ser utilizado tanto para la detección de vehículos, la determinación de su velocidad o su tipología como para la generación de energía eléctrica para su posterior almacenamiento.

\subsection{Cable piezoeléctrico}

Uno de los desarrollos industriales más recientes en la tecnología de polímeros piezoeléctricos es su presentación en forma de cable, cuya estructura es la de un cable coaxial siendo el aislante interno un copolímero piezoeléctrico generalmente del tipo TrFE o de PVDF, polímero ferro-piezoeléctrico, tal y como se aprecia en la figura 3.

Para comprender el comportamiento de los cables piezoeléctricos utilizados en las aplicaciones descritas anteriormente y diseñar los circuitos de detección que acondicionan y procesan las señales generadas, se debe

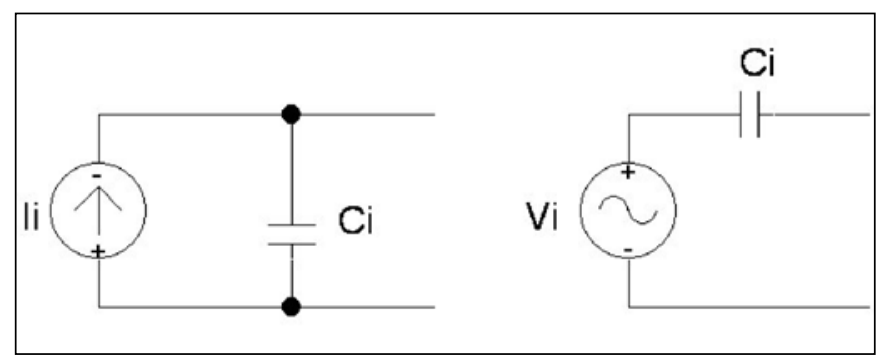

Figura 4. Equivalentes eléctricos del cable piezoeléctrico en aplicaciones de conversión de energía mecánica a eléctrica. Fuentes
TABLA II. CARACTERÍSTICAS TÍPICAS DE CABLE PIEZOELÉCTRICO COMERCIAL

\begin{tabular}{|l|c|c|c|}
\hline Propiedades Típicas & Unidades & $\begin{array}{c}\text { Cable } \\
\text { Espiral }\end{array}$ & $\begin{array}{c}\text { Cable } \\
\text { copolímero }\end{array}$ \\
\hline Diámetro exterior & $\mathrm{mm}$ & 2,69 & 2,72 \\
\hline Capacidad (1kHz) & $\mathrm{pF} / \mathrm{m}$ & 950 & 655 \\
\hline Peso & $\mathrm{Kg} / \mathrm{km}$ & 14,5 & 15,5 \\
\hline Resistencia (escudo) & $\mathrm{DCR} / \mathrm{km}$ & 47 & 47 \\
\hline Tangente delta & $(1 \mathrm{kHz})$ & 0,016 & 0,017 \\
\hline Coeficiente Hidrostático & $\mathrm{pC} / \mathrm{N}$ & 20 & 15 \\
\cline { 2 - 5 } Piezoeléctrico & $\mathrm{Vm} / \mathrm{N}$ & & $150{ }^{*} 10^{-3}$ \\
\hline Resistencia (centro) & $\mathrm{DCR} / \mathrm{km}$ & 31 & 31 \\
\hline
\end{tabular}

modelar eléctricamente al cable utilizando su equivalente como fuente de tensión o su transformación Norton como fuente de corriente, en asociación con el elemento capacitivo tal y como se presenta en la figura 4, así las ecuaciones que rigen el comportamiento del cable se pueden simplificar, aplicando las restricciones geométricas a las siguientes:

Tensión en circuito abierto, siendo t el espesor del aislante piezoeléctrico.

$$
V=g_{3 n} X_{n} t
$$

Densidad de carga generada en condiciones próximas a cortocircuito, con $X_{n}$ la presión $\left(\mathrm{N} / \mathrm{m}^{2}\right)$ en el eje $n$.

$$
D=d_{3 n} X_{n}
$$

En la tabla II, se reproducen las características típicas facilitadas por el fabricante Measurement Specialties del cable con el que se han realizado los ensayos descritos en el artículo. La información que se facilita resulta insuficiente para modelar el generador equivalente de la figura 4 que reproduzca exactamente el comportamiento del cable en las aplicaciones relacionadas con tráfico rodado. Será a partir del análisis detallado de las medidas tomadas en un entorno real, como se construirá el modelo eléctrico exacto que complemente la información facilitada, al poder introducir los efectos no lineales que finalmente reproducen el comportamiento de los cables piezoeléctricos.

\subsection{Utilización en aplicaciones viales}

El cable piezoeléctrico se encuentra en aplicaciones relacionadas con la gestión y supervisión del tráfico en vías de comunicación para automoción. Su instalación no requiere grandes modificaciones en la calzada ya que se introduce en el asfalto a escasos centímetros de la superficie.

Se han realizado ensayos sobre cables piezoeléctricos, disponiendo los mismos siguiendo los correspondientes diámetros de la plataforma estática, dado que bajo el conjunto motor-reductor que ocupa la parte central del banco, se ha 
mecanizado un canal que permite pasar el cable objeto de ensayo para que el impacto lo reciban las dos secciones que se ofrecen al paso de las ruedas de cada eje. Adicionalmente, se ha realizado un canal a $90^{\circ}$ del primero, para conducir el cable utilizado para sensar la velocidad real de la plataforma, medida necesaria para el funcionamiento del control a velocidad constante del banco de ensayos con independencia de las condiciones inerciales de la plataforma.

La información facilitada por el fabricante del cable piezoeléctrico ( $\mathrm{P} / \mathrm{N}$ : 1005801-1 de Measurement Specialties, Inc.) utilizado en los ensayos efectuados en el laboratorio, para aplicaciones en las que el cable se encuentra enterrado, es excesivamente escueta al no facilitar las condiciones exactas de medida, ya que no se detalla ni las características del equipo de medida (resulta fundamental conocer su impedancia de entrada), ni el tipo de vehículo automóvil con el que se han registrado las gráficas. No obstante, se aprecian valores orientativos del orden de magnitud para las amplitudes esperadas y separación en el tiempo entre los pasos de ambos ejes de un vehículo de tipo automóvil.

\subsection{Ensayos a baja velocidad}

En la figura 5(a) se recogen las medidas tomadas en el osciloscopio TDS7054 de Agilent Technologies, con sonda de prueba $10073 \mathrm{C}$ ( 10:1, $500 \mathrm{MHz} \mathrm{BW})$, seleccionando $1 \mathrm{M} \Omega$ como impedancia de entrada. La disposición de los ejes de rodadura se encuentran separados un ángulo de $90^{\circ}$, permitiendo así cubrir ensayos a velocidades comprendidas entre las posiciones $7^{\mathrm{a}}$ y $9^{\mathrm{a}}$ recogidas en la tabla I . El valor de la impedancia de entrada del equipo de medida resulta muy importante, ya que el equivalente eléctrico del cable piezoeléctrico es el de una fuente de alta impedancia de salida, al presentar la capacidad del cable un valor muy pequeño, en nuestros ensayos se dispone de cable de 3 metros de longitud, con lo que su capacidad resulta ser de 2,85 nF atendiendo a la tabla II, donde se proporcionan los parámetros que ofrece el fabricante, lo que unido a las bajas frecuencias de excitación correspondientes a las aplicaciones viales, conllevan a que la impedancia terminal del sistema de medida sea determinante para justificar las amplitudes recogidas.
Se puede inferir rápidamente de los resultados recogidos que el cálculo de la velocidad equivalente (expresada en $\mathrm{km} / \mathrm{h}$ ) resulta de aplicar la ecuación siguiente:

$$
v=\frac{b}{t_{e}} \cdot 3,6
$$

Dónde b representa la cota de batalla del automóvil (expresada en $\mathrm{m}$ ) y $\mathrm{t}_{\mathrm{e}}$ el tiempo entre los impactos registrados entre ejes ( expresado en s. ). Así, para la diferencia entre impactos recogida en el ensayo que reproduce la figura 5(a) de $538 \mathrm{~ms}$, se corresponderá para un automóvil de tipo medio, cuya distancia entre ejes sea de $b=2,64 \mathrm{~m}$, con una velocidad en carretera de $17,665 \mathrm{~km} / \mathrm{h}$.

La amplitud de pico máxima en valor positivo resulta ser de $900 \mathrm{mV}$, así como el valor instantáneo de pico negativo registrado fue de $-993,75 \mathrm{mV}$. La disposición de los ejes tal y como se ha comentado fue de $90^{\circ}$ y no se ha suplementado masa adicional.

En la figura 5(b) se recoge el resultado obtenido para una disposición de ejes a $90^{\circ}$, sin masa adicional aplicada y una consigna de velocidad equivalente de $29,333 \mathrm{~km} / \mathrm{h}$.

\subsection{Ensayos a velocidades altas}

Realizando el cambio de un eje de rodadura a la posición de $\beta=22^{\circ}$ respecto al eje fijo, se alcanzan los mayores valores de velocidades equivalentes, tal y como recoge la tabla I para el rango correspondiente a las posiciones $1^{\mathrm{a}}, 2^{\mathrm{a}}$ y $3^{\mathrm{a}}$ de dicha tabla.

En la figura 5 (c) se recoge la medida capturada con una consigna de velocidad de $\mathrm{v}=69,474 \mathrm{~km} / \mathrm{h}$.

En la figura 6 se presenta el panel de control realizado en LabVIEW ${ }^{\mathrm{TM}}$ del banco de ensayos durante la realización de una prueba para el mismo tipo de vehículo automóvil anteriormente descrito a $120 \mathrm{~km} / \mathrm{h}$ (tiempo entre ejes, $t_{\mathrm{e}}=$ 0,0792 s), máxima velocidad autorizada en vías rápidas en España. Esta aplicación recoge de forma indexada los datos identificativos de cada prueba, así como los datos de las formas de onda medidas, en formato gráfico y de texto, para

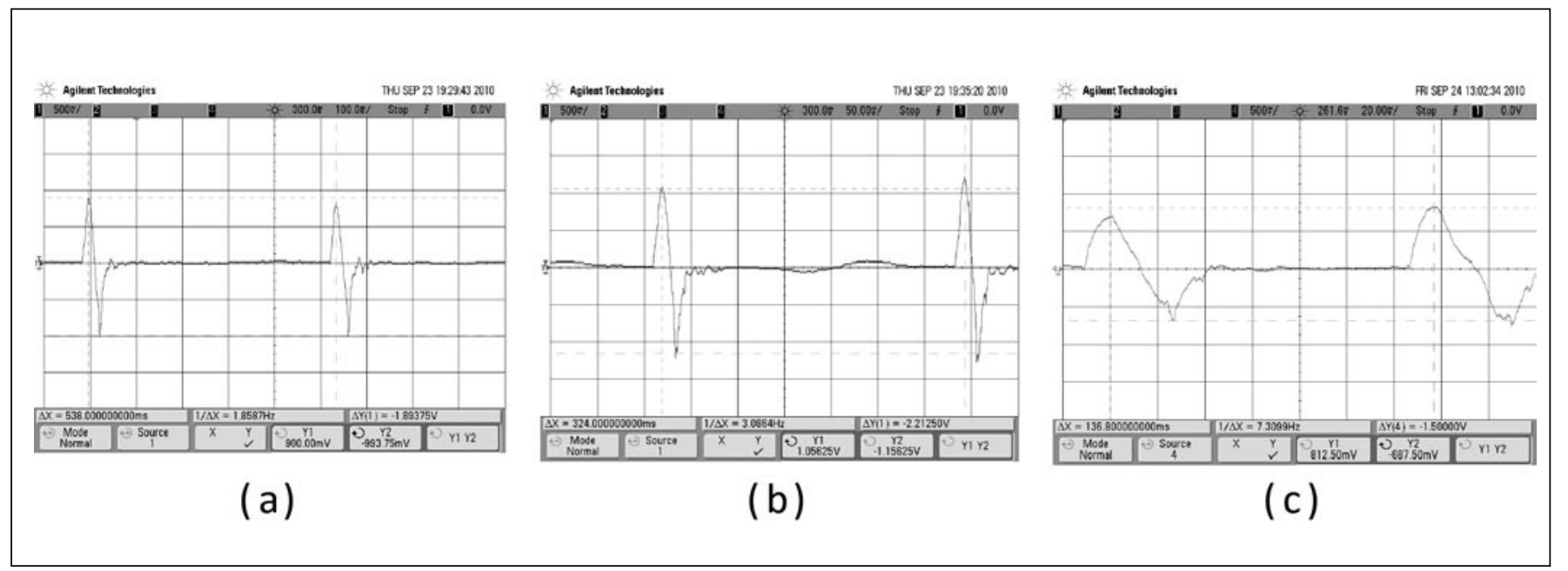

Figura 5: Ensayos a distintas velocidades: (a) 17,665 km/h. (b) 29,333 km/h y (c) 69,474 km/h 
su posterior análisis. Asimismo, se representan en pantalla la velocidad del banco de ensayos expresada en $\mathrm{km} / \mathrm{h}$ resultante para un vehículo cuya distancia entre ejes se haya introducido previamente a la aplicación, así como la forma de onda del estímulo de tensión recogido en el cable sometido a ensayo, mostrándose el paso de dos vehículos consecutivos. Como se ha comentado anteriormente, la medida exacta de la velocidad equivalente presentada en la figura 6 , se obtiene mediante el programa escrito en LabVIEW ${ }^{\mathrm{TM}}$ que procesa la señal acondicionada sensada mediante un cable piezoeléctrico dedicado.

\subsection{Respuesta con masa suplementaria y amplificación mecánica}

Para la realización de ensayos dónde se suplementa la masa que se aplica a cada eje, solamente se necesita disponer de masas de valor conocido sobre cada rueda y sobre la zona central del eje de rotación. En los ensayos efectuados sobre cuatro cables simultáneamente, se han utilizado pesas deportivas de $15 \mathrm{~kg}$ y de $10 \mathrm{~kg}$ en diversas combinaciones. En la figura 7(a) se recogen resultados de medida para un ensayo efectuado con un suplemento total de $160 \mathrm{~kg}$ en uno de los ejes. En la figura 7(b) se presentan los resultados para otra prueba donde se ha aplicado amplificación mecánica del estímulo de presión.

Estos ensayos se aplican para reproducir los estímulos que recogen los cables piezoeléctricos enterrados al paso de vehículos pesados.

\section{CONCLUSIONES}

El diseño original del banco de ensayos realizado nos proporciona una nueva herramienta robusta, automatizada y versátil, útil para reproducir el comportamiento de los materiales piezoeléctricos en aplicaciones relacionadas con el tráfico vial, donde típicamente se encuentran en forma de cable enterrado. Los resultados experimentales recogidos tanto

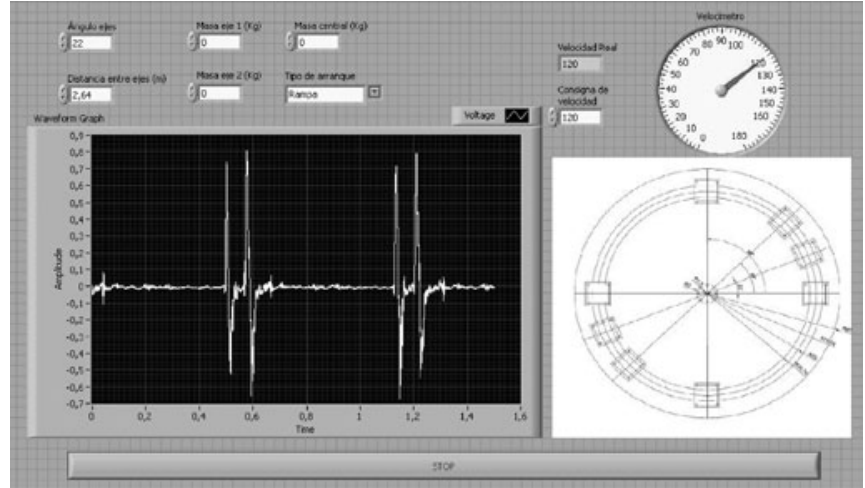

Figura 6: Aplicación software de control del banco de ensayos y de recogida de resultados desarrollada en LabVIEW ${ }^{\mathrm{TM}}$ durante un ensayo a $120 \mathrm{~km} / \mathrm{h}$

en osciloscopio como mediante un sistema de adquisición de datos basado en LabView ${ }^{\mathrm{TM}}$ de National Instruments ${ }^{\mathrm{TM}}$, permiten reproducir el comportamiento de estos materiales en la realidad, cubriéndose en los ensayos un amplio rango de velocidades para diversos tipos de vehículos. El análisis de los ensayos registrados permite conocer datos relacionados con su comportamiento o la fatiga que puedan experimentar en su uso real. De este análisis, se realizará un modelo eléctrico equivalente robusto que recoja tanto el efecto de la variación de la velocidad como del estímulo mecánico aplicado, modelo necesario para abrir la investigación hacia las aplicaciones relativas al aprovechamiento energético de la energía eléctrica generada en los mismos.

\section{AGRADECIMIENTOS}

Este trabajo se ha financiado con los proyectos MAT200766845-C02-02, MAT2010-21088-C03-03 y la acción CAM-UPM 139/Q06 0915-110

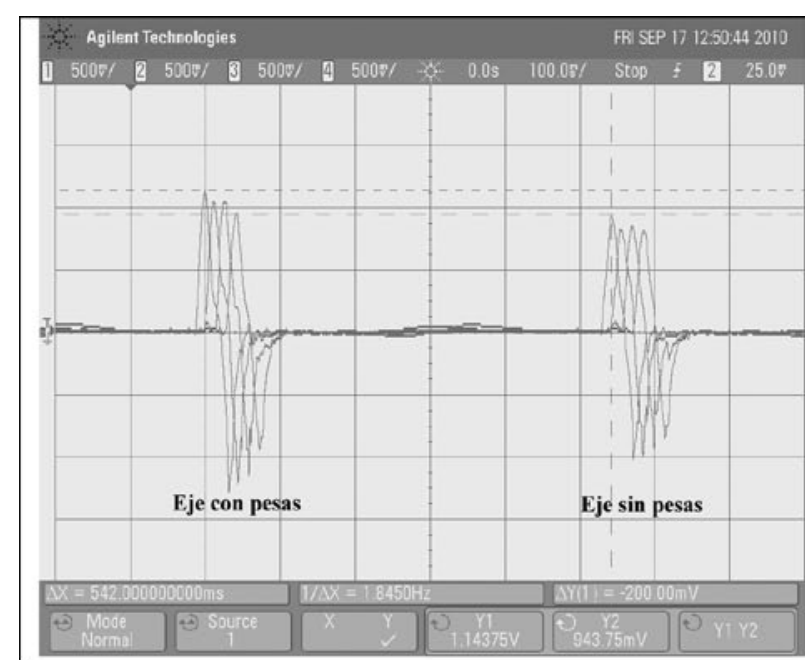

(a)

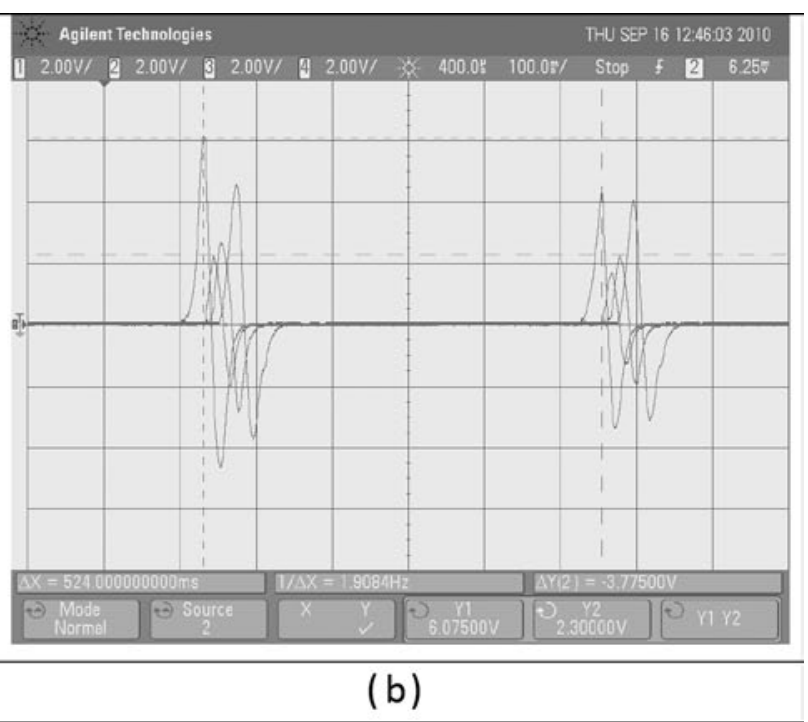

(b)

Figura 7: Ensayo efectuado sobre cuatro cables piezoeléctricos (a)con suplemento de 160 kg en un eje y (b) con amplificación mecánica. 


\section{BIBLIOGRAFÍA}

1. R Lahtinen, T. Muukkonen, Koskinen. A piezopaint-based sensor for monitoring structure dynamics, J. Smart Materials \& Structures 16(6) 25712576 (2007)

2. ZX. Li, XM. Yang, ZJ. Li. Application of cement-based piezoelectric sensors for monitoring traffic flows. J. Transp. Eng ASCE 132 (7) 565-573 (2006)

3. J. Iaquinta, E. Merliot, L.M. Cottineau. Piezoelectric sensors for weigh-inmotion systems: J. Testing And Evaluation 32 (6): 476-483 (2004)

4. H. A. Sodano, D. J. Inman, G. Park. A Review of Power Harvesting from Vibration using Piezoelectric Materials. The Shock Vibration Digest, 36 (3), 197-205, (2004)

5. H. A. Sodano, D. J. Inman, G. Park. Generation and Storage of Electricity from Power Harvesting Devices. J. of Intelligent Mat. Syst. and Struct. 16(1) 67-75 (2005)
6. K. Hyeoungwoo, P. Shashank, H. Stephanou, K. Uchino. Consideration of Impedance Matching Techniques for Efficient Piezoelectric Energy Harvesting IEEE UFFC 54(9) 1851 (2007)

7. F.J. Jiménez, de Frutos J. Noncontact inspection laser system for characterization of piezoelectric samples, Review Scientific Instruments, 75, 4497-4504 (2004)

8. F.J. Jiménez, de Frutos J. Virtual instrument for measurement, processing data, and visualization of vibration patterns of piezoelectric devices, computer standards \& interfaces, 27, 653-663 (2005)

9. AlvarezArenas G, Gonzalez AM, DeFrutos J, deEspinosa FRM, Piezoelectric characterisation of porous piezoceramics, IEEE ULTRASONICS SYMPOSIUM, PROCEEDINGS, VOLS 1-2 : 519-522 (1996)

10. AM. Gonzalez, J. De Frutos, C. Duro, C. Alemany, L. Pardo, Changes in the piezoelectric parameters of PZT ceramics during the poling process, FERROELECTRICS 208 1-4 : 449-457 (1998)

Recibido: $17 / 11 / 2010$

Aceptado: 15/03/2011

\section{FE DE ERRATAS}

En el anterior número del Boletín de la Sociedad Española de Cerámica y Vidrio se intercambiaron por error los códigos ISSN de las ediciones en papel y electrónica, siendo CORRECTOS

los códigos ISSN siguientes:

- Para edición en papel: ISSN 0366-3175

- Para edición electrónica: eISSN 2173-0431 